
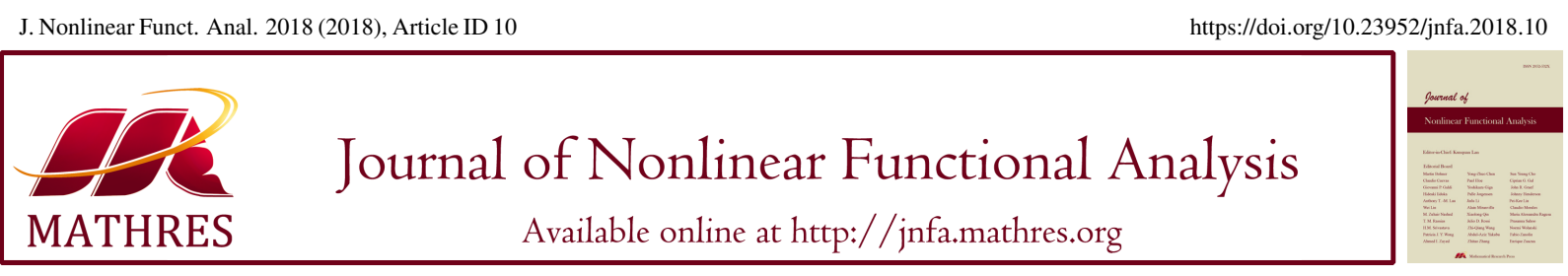

\title{
ON $p(x)$-KIRCHHOFF EQUATIONS WITH CONCAVE-CONVEX TERMS IN UNBOUNDED DOMAINS
}

\author{
MOHAMMED MASSAR ${ }^{1, *}$, MOHAMED TALBI $^{2}$, NAJIB TSOULI $^{3}$, HAFID LEBRIMCHI $^{3}$ \\ ${ }^{1}$ Department of Mathematics, Mohamed I University, FSTH, Morocco \\ ${ }^{2}$ CRMEF, Oujda, Morocco \\ ${ }^{3}$ Department of Mathematics, Mohamed I University, FSO, Morocco
}

\begin{abstract}
In this paper, we study the existence of solutions for a class of $p(x)$-Kirchhoff problems in unbounded domains with concave-convex terms. The technical approach in this paper is mainly based on the mountain pass theorem and Ekeland's variational principle.
\end{abstract}

Keywords. $p(x)$-Laplacian; Kirchhoff equation; Concave-convex term; Ekeland's variational principle.

2010 Mathematics Subject Classification. 35J35, 35J60, 35J92.

\section{INTRODUCTION}

In this article, we are concerned with the following problem

$$
\begin{cases}L(u)=\lambda f(x)|u|^{q(x)-2} u+g(x)|u|^{r(x)-2} u+h(x) & \text { in } \Omega, \\ \frac{\partial u}{\partial v}=0 & \text { on } \partial \Omega,\end{cases}
$$

where $L(u):=-M\left(\int_{\Omega} \frac{|\nabla u|^{p(x)}+\mid u^{p(x)}}{p(x)} d x\right)\left(\Delta_{p(x)} u-|u|^{p(x)-2} u\right), \lambda>0, \Omega=\mathbb{R}^{N} \backslash \overline{\Omega_{b}}$ with $\Omega_{b} \subset \mathbb{R}^{N}$ is a smooth bounded domain, $M$ is a positive continuous function on $[0,+\infty), g$ is a nonnegative continuous function, $f$ and $h$ are continuous functions which may change sign on $\Omega$ and $p, q, r \in C_{+}(\bar{\Omega})$, where

$$
C_{+}(\bar{\Omega})=\left\{\sigma \in C(\bar{\Omega}) \cap L^{\infty}(\Omega): \inf _{x \in \bar{\Omega}} \sigma(x)>1\right\} .
$$

Moreover $p$ is Lipschitz continuous, $1<p^{-}:=\inf _{x \in \Omega} p(x) \leq p^{+}:=\sup _{x \in \Omega} p(x)<N$ and $r(x)<p^{*}(x), \forall x \in \bar{\Omega}$.

The study of various problems with nonstandard growth conditions has been received considerable attention because of their large applications in elastic mechanics electrorheological fluids, image restoration, dielectric breakdown, electrical resistivity and polycrystal plasticity and continuum mechanics; see

\footnotetext{
${ }^{*}$ Corresponding author.

E-mail addresses: massarmed@ hotmail.com (M. Massar), talbijdi@gmail.com (M. Talbi), tsouli@hotmail.com (N. Tsouli), hafid_lebrimchi@yahoo.fr (H. Lebrimchi).

Received June 13, 2017; Accepted February 10, 2018.
}

(C)2018 Journal of Nonlinear Functional Analysis 
$[1,2,3,4,5,6,7]$ and the references therein. Elliptic problems of Kirchhoff-type involving operators like the $p(x)$-Laplacian or its generalizations have been the object of ever more attention in these latest years; see, for instance, $[8,9,10,11,12,13,14,15]$ and the references therein. However, to our knowledge, there are not many papers $[16,17,18]$ which dealt with nonlocal $p$-Kirchhoff equations with concave-convex terms.

Motivated by the above mentioned works, we will use the Mountain Pass theorem and Ekeland's variational principle to prove the existence of two solutions for problem $\left(P_{\lambda}\right)$ in unbounded domains with nonhomogeneous term $h(x)$.

The assumptions under problem $\left(P_{\lambda}\right)$ will be considered are the following:

$\left(M_{1}\right)$ There exist $m_{1} \geq m_{0}>0$ and $\frac{q^{+}}{p^{-}}<\alpha \leq \beta<\frac{r^{-}}{p^{+}}$such that

$$
m_{0} t^{\alpha} \leq \widehat{M}(t):=\int_{0}^{t} M(s) d s \leq m_{1} t^{\beta} \text { for all } t>0 ;
$$

$\left(M_{2}\right)$ There exists $0<\theta<\frac{r^{-}}{p^{+}}$such that

$$
\theta \widehat{M}(t) \geq M(t) t \text { for all } t>T_{0}>0
$$

$\left(H_{0}\right) f \in L^{\infty}(\Omega) \cap L^{q_{0}(x)}(\Omega)$, with $q_{0}(x)=\frac{p^{*}(x)}{p^{*}(x)-q(x)} \forall x \in \bar{\Omega}$;

$\left(H_{1}\right) \quad 0 \leq g \in L^{\infty}(\Omega) \cap L^{r_{0}(x)}(\Omega)$, with $r_{0}(x)=\frac{p^{*}(x)}{p^{*}(x)-r(x)} \forall x \in \bar{\Omega}$;

$\left(H_{2}\right) h \in L^{\infty}(\Omega) \cap L^{s_{0}(x)}(\Omega)$, with $s_{0}(x)=\frac{p^{*}(x)}{p^{*}(x)-1} \forall x \in \bar{\Omega}$.

\section{PRELIMINARIES AND MAIN RESUlT}

In this section, we recall some interesting properties of the variable exponent Lebesgue and Sobolev spaces that will be used to study problem $\left(P_{\lambda}\right)$. Let $\Omega \subset \mathbb{R}^{N}$ an open domain. Define the variable exponent Lebesgue space by

$$
L^{p(x)}(\Omega)=\left\{u: \Omega \rightarrow \mathbb{R} \text { measurable }: \int_{\Omega}|u(x)|^{p(x)} d x<\infty\right\} .
$$

This space endowed with the Luxemburg norm,

$$
\|u\|_{L^{p(x)}(\Omega)}=\inf \left\{\tau>0: \int_{\Omega}\left|\frac{u(x)}{\tau}\right|^{p(x)} d x \leq 1\right\}
$$

is a separable and reflexive Banach space. Denoting by $L^{p^{\prime}(x)}(\Omega)$ the conjugate space of $L^{p(x)}(\Omega)$ where $\frac{1}{p(x)}+\frac{1}{p^{\prime}(x)}=1$ for any $u \in L^{p(x)}(\Omega)$ and $v \in L^{p^{\prime}(x)}(\Omega)$, we have the following Hölder type inequality

$$
\int_{\Omega}|u v| d x \leq\left(\frac{1}{p^{-}}+\frac{1}{p^{\prime-}}\right)\|u\|_{L^{p(x)}(\Omega)}\|v\|_{L^{p^{\prime}(x)}(\Omega)} .
$$

Now, we introduce the modular of the Lebesgue-Sobolev space $L^{p(x)}(\Omega)$ as the mapping $\rho_{p(x)}: L^{p(x)}(\Omega) \rightarrow$ $\mathbb{R}$, defined by

$$
\rho_{p(x)}(u)=\int_{\Omega}|u|^{p(x)} d x, \forall u \in L^{p(x)}(\Omega) .
$$

The relation between modular and Luxemburg norm is clarified by the following proposition: 
Proposition 2.1 ([19]). If $u, u_{n} \in L^{p(x)}(\Omega)$, then following properties hold:

(1) $\|u\|_{L^{p(x)}(\Omega)} \leq 1 \Rightarrow\|u\|_{L^{p(x)}(\Omega)}^{p^{+}} \leq \rho_{p(x)}(u) \leq\|u\|_{L^{p(x)}(\Omega)}^{p^{-}} ;$

(2) $\|u\|_{L^{p(x)}(\Omega)} \geq 1 \Rightarrow\|u\|_{L^{p(x)}(\Omega)}^{p^{-}} \leq \rho_{p(x)}(u) \leq\|u\|_{L^{p(x)}(\Omega)}^{p^{+}}$;

(3) $\lim _{n \rightarrow \infty}\left\|u_{n}-u\right\|_{L^{p(x)}(\Omega)}=0 \Leftrightarrow \lim _{n \rightarrow \infty} \rho_{p(x)}\left(u_{n}-u\right)=0$.

Next, we define the variable exponent Sobolev space $W^{1, p(x)}(\Omega)$ by

$$
W^{1, p(x)}(\Omega)=\left\{u \in L^{p(x)}(\Omega):|\nabla u| \in L^{p(x)}(\Omega)\right\},
$$

endowed with the norm

$$
\|u\|=\|u\|_{L^{p(x)}(\Omega)}+\|\nabla u\|_{L^{p(x)}(\Omega)} .
$$

$W^{1, p(x)}(\Omega)$ is a Banach space which is reflexive under condition $1<p^{-} \leq p^{+}<+\infty$. If $q \in C_{+}(\bar{\Omega})$ and $p(x) \leq q(x) \leq p^{*}(x) \forall x \in \bar{\Omega}$, then the embedding $W^{1, p(x)}(\Omega) \hookrightarrow L^{q(x)}(\Omega)$ is continuous. This last embedding is compact when $\Omega$ is bounded and that $q(x)<p^{*}(x) \forall x \in \bar{\Omega}$. Setting

$$
\rho_{1, p(x)}(u)=\int_{\Omega}\left(|\nabla u|^{p(x)}+|u|^{p(x)}\right) d x, \forall u \in W^{1, p(x)}(\Omega),
$$

similarly to Proposition 2.1, it holds:

Proposition 2.2 ([19]). If $u, u_{n} \in W^{1, p(x)}(\Omega)$, then following properties hold true:

(1) $\|u\| \leq 1 \Rightarrow\|u\|\left\|^{p^{+}} \leq \rho_{1, p(x)}(u) \leq\right\| u \|^{p^{-}}$;

(2) $\|u\| \geq 1 \Rightarrow\|u\|\left\|^{p^{-}} \leq \rho_{1, p(x)}(u) \leq\right\| u \|^{p^{+}}$;

(3) $\lim _{n \rightarrow \infty}\left\|u_{n}-u\right\|=0 \Leftrightarrow \lim _{n \rightarrow \infty} \rho_{1, p(x)}\left(u_{n}-u\right)=0$.

Definition 2.3. We say that $u \in W^{1, p(x)}(\Omega)$ is a weak solution of problem $\left(P_{\lambda}\right)$ if

$$
\begin{aligned}
& M\left(\int_{\Omega} \frac{|\nabla u|^{p(x)}+|u|^{p(x)}}{p(x)} d x\right) \int_{\Omega}\left(|\nabla u|^{p(x)-2} \nabla u \nabla v+|u|^{p(x)-2} u v\right) d x \\
& -\lambda \int_{\Omega} f(x)|u|^{q(x)-2} u v d x-\int_{\Omega} g(x)|u|^{r(x)-2} u v d x-\int_{\Omega} h(x) v d x=0,
\end{aligned}
$$

for all $v \in W^{1, p(x)}(\Omega)$.

Our main result is the following theorem.

Theorem 2.4. Assume that $\left(M_{1}\right)-\left(M_{2}\right)$ and $\left(H_{0}\right)-\left(H_{2}\right)$ hold. Then, there exist $\lambda_{0}, \mu>0$ such that for all $\lambda \in\left(0, \lambda_{0}\right)$, problem $\left(P_{\lambda}\right)$ has at least two nontrivial weak solutions provided that $\|h\|_{L^{s_{0}(x)}(\Omega)} \leq \mu$.

\section{PROOF OF MAIN RESUlT}

Since we will rely on the critical point theory, we define the energy functional corresponding to prob$\operatorname{lem}\left(P_{\lambda}\right)$ as $I_{\lambda}: W^{1, p(x)}(\Omega) \mapsto \mathbb{R}$,

$$
I_{\lambda}(u)=\widehat{M}\left(\int_{\Omega} \frac{|\nabla u|^{p(x)}+|u|^{p(x)}}{p(x)} d x\right)-\lambda \int_{\Omega} f(x) \frac{|u|^{q(x)}}{q(x)} d x-\int_{\Omega} g(x) \frac{|u|^{r(x)}}{r(x)} d x-\int_{\Omega} h(x) u d x .
$$

Tacking account that $M$ is continue on $[0,+\infty)$, by assumptions $\left(H_{0}\right)-\left(H_{2}\right)$ and standard arguments, we see that $I_{\lambda} \in C^{1}\left(W^{1, p(x)}(\Omega), \mathbb{R}\right)$ (see for example, [20, 21, 22]. Moreover, the critical points of $I_{\lambda}$ are weak solutions of problem $\left(P_{\lambda}\right)$. 
Lemma 3.1. Assume that $\left(M_{1}\right)-\left(M_{2}\right)$ and $\left(H_{0}\right)-\left(H_{2}\right)$ hold. Then there exist $\lambda_{0}, \mu, \rho, \kappa>0$ such that for $\lambda \in\left(0, \lambda_{0}\right)$ and $\|h\|_{L^{s_{0}(x)}(\Omega)} \leq \mu$,

$$
I_{\lambda}(u) \geq \kappa \text { for all }\|u\|=\rho .
$$

Moreover, there exists $e \in W^{1, p(x)}(\Omega)$ with $\|e\|>\rho$, such that $I_{\lambda}(e)<0$.

Proof. From $\left(H_{0}\right)$, Hölder's inequality and Proposition 2.1, we have

$$
\begin{aligned}
\int_{\Omega}|f(x) \| u|^{q(x)} d x & \leq c_{0}\|f\|_{L^{q_{0}(x)}(\Omega)}\left|\left\|\left.u\right|^{q(x)}\right\|_{L^{p^{*}(x)}}\right. \\
& \leq c_{0}\|f\|_{L^{q_{0}(x)}(\Omega)} \max \left(\|u\|_{L^{p^{*}(x)}(\Omega)}^{q^{+}},\|u\|_{L^{p^{*}(x)}(\Omega)}^{q^{-}}\right) \\
& \leq c_{1}\|f\|_{L^{q_{0}(x)}(\Omega)} \max \left(\left.\|u\|\right|^{q^{+}},\left.\|u\|\right|^{q^{-}}\right) .
\end{aligned}
$$

Similarly, one has

$$
\int_{\Omega} g(x)|u|^{r(x)} d x \leq c_{2}\|g\|_{L^{r_{0}(x)}(\Omega)} \max \left(\|u\|^{r^{+}},\|u\|^{r^{-}}\right)
$$

and

$$
\int_{\Omega}\left|h(x)\left\|u \mid d x \leq c_{3}\right\| h\left\|_{L^{s_{0}(x)}(\Omega)}\right\| u \| .\right.
$$

On the other hand, using Young's inequality, for given $\varepsilon>0$, there exists $C_{\varepsilon}$ such that

$$
\|h\|_{L^{s_{0}(x)}(\Omega)}\|u\| \leq \varepsilon\|u\|^{\alpha p^{+}}+C_{\varepsilon}\|h\|_{L^{s_{0}(x)}(\Omega)}^{\frac{\alpha p^{+}}{\alpha p^{+}-1}} .
$$

By $\left(M_{1}\right),(3.1)-(3.4)$ and Proposition 2.2, for $\|u\|<1$, we have

$$
\begin{aligned}
I_{\lambda}(u) \geq & m_{0}\left(\int_{\Omega} \frac{|\nabla u|^{p(x)}+|u|^{p(x)}}{p(x)} d x\right)^{\alpha}-\frac{\lambda}{q^{-}} \int_{\Omega}|f(x) \| u|^{q(x)} d x \\
& -\frac{1}{r^{-}} \int_{\Omega} g(x)|u|^{r(x)} d x-\int_{\Omega}|h(x) \| u| d x \\
\geq & \frac{m_{0}}{\left(p^{+}\right)^{\alpha}}\|u\|^{\alpha p^{+}}-\frac{\lambda c_{1}}{q^{-}}\|f\|_{L^{q_{0}(x)}(\Omega)}\|u\|^{q^{-}}-\frac{c_{2}}{r^{-}}\|g\|_{L^{r_{0}(x)}(\Omega)}\|u\|^{r^{-}} \\
& -c_{3} \varepsilon\|u\|^{\alpha p^{+}}-c_{3} C_{\varepsilon}\|h\|_{L^{s_{0}(x)}(\Omega)}^{\frac{\alpha p^{+}}{\alpha^{+}-1}} .
\end{aligned}
$$

Choosing $\varepsilon=\frac{m_{0}}{2 c_{3}\left(p^{+}\right)^{\alpha}}$, we obtain

$$
\begin{aligned}
I_{\lambda}(u) \geq & \frac{m_{0}}{2\left(p^{+}\right)^{\alpha}}\|u\|^{\alpha p^{+}}-\frac{\lambda c_{1}}{q^{-}}\|f\|_{L^{q_{0}(x)}(\Omega)}\|u\|^{q^{-}}-\frac{c_{2}}{r^{-}}\|g\|_{L^{r_{0}(x)}(\Omega)}\|u\|^{r^{-}}-c_{3} C_{\varepsilon}\|h\|_{L^{s_{0}(x)}(\Omega)}^{\frac{\alpha p^{+}}{\alpha p^{+}}} \\
= & \|u\|^{\alpha p^{+}}\left(\frac{m_{0}}{2\left(p^{+}\right)^{\alpha}}-\frac{\lambda c_{1}}{q^{-}}\|f\|_{L^{q_{0}(x)}(\Omega)}\|u\|^{q^{-}-\alpha p^{+}}-\left.\frac{c_{2}}{r^{-}}\|g\|_{L^{r_{0}(x)}(\Omega)}\|u\|\right|^{r^{-}-\alpha p^{+}}\right) \\
& -c_{3} C_{\varepsilon}\|h\|_{L^{s_{0}(x)}(\Omega)}^{\frac{\alpha p^{+}}{\alpha p^{+}-1}} .
\end{aligned}
$$

Let

$$
\gamma(\tau)=a_{0} \lambda \tau^{q^{-}-\alpha p^{+}}+a_{1} \tau^{r^{-}-\alpha p^{+}}, 0<\tau<1,
$$

where $a_{0}=\frac{c_{1}}{q^{-}}\|f\|_{L^{q_{0}(x)}(\Omega)}, a_{1}=\frac{c_{2}}{r^{-}}\|g\|_{L^{r_{0}(x)}(\Omega)}$. Since $q^{-}<\alpha p^{+}$, we see that $\gamma(\tau) \rightarrow+\infty$ as $\tau \rightarrow 0^{+}$. Then $\gamma$ has a minimum at

$$
\tau_{0}:=\left(\frac{a_{0} \lambda\left(\alpha p^{+}-q^{-}\right)}{a_{1}\left(r^{-}-\alpha p^{+}\right)}\right)^{1 /\left(r^{-}-q^{-}\right)} .
$$


Moreover

$$
\gamma\left(\tau_{0}\right)=\frac{a_{0}\left(r^{-}-q^{-}\right)}{r^{-}-\alpha p^{+}}\left(\frac{a_{0}\left(\alpha p^{+}-q^{-}\right)}{a_{1}\left(r^{-}-\alpha p^{+}\right)}\right)^{\left(q^{-}-\alpha p^{+}\right) /\left(r^{-}-q^{-}\right)} \lambda^{\left(r^{-}-\alpha p^{+}\right) /\left(r^{-}-q^{-}\right)} \underset{\lambda \rightarrow 0^{+}}{\longrightarrow} 0 .
$$

Thus, we can find $\lambda_{0}>0$ such that $\gamma\left(\tau_{0}\right)<\frac{m_{0}}{2\left(p^{+}\right)^{\alpha}} \forall \lambda \in\left(0, \lambda_{0}\right)$. From (3.5), for $\|u\|=\tau_{0}$, we have

$$
I_{\lambda}(u) \geq \tau_{0}^{\alpha p^{+}}\left(\frac{m_{0}}{2\left(p^{+}\right)^{\alpha}}-\gamma\left(\tau_{0}\right)\right)-c_{3} C_{\mathcal{E}}\|h\|_{L^{s_{0}(x)}(\Omega)}^{\frac{\alpha p^{+}}{\alpha p^{+}}}
$$

Set

$$
\kappa:=\tau_{0}^{\alpha p^{+}}\left(\frac{m_{0}}{2\left(p^{+}\right)^{\alpha}}-\gamma\left(\tau_{0}\right)\right)-c_{3} C_{\varepsilon}\|h\|_{L^{s_{0}(x)}(\Omega)}^{\frac{\alpha^{+}}{\alpha p^{+}}}
$$

and

$$
\mu:=\left[\frac{\tau_{0}^{\alpha p^{+}}}{c_{3} C_{\varepsilon}}\left(\frac{m_{0}}{2\left(p^{+}\right)^{\alpha}}-\gamma\left(\tau_{0}\right)\right)\right]^{\frac{\alpha p^{+}-1}{\alpha p^{+}}}
$$

It follows from (3.6) that for each $\lambda \in\left(0, \lambda_{0}\right)$ and $\|h\|_{L^{s_{0}(x)}(\Omega)}<\mu$,

$$
I_{\lambda}(u) \geq \kappa>0 \text { for }\|u\|=\rho=\tau_{0}
$$

Now, let $\varphi_{0} \in C_{0}^{\infty}\left(\Omega_{0}\right)$, where $\Omega_{0} \subset\{x \in \Omega: g(x)>0\}$. In view of $\left(M_{1}\right)$, for $t$ large enough

$$
\begin{aligned}
I_{\lambda}\left(t \varphi_{0}\right)= & \widehat{M}\left(\int_{\Omega} \frac{\left|\nabla t \varphi_{0}\right|^{p(x)}+\left|t \varphi_{0}\right|^{p(x)}}{p(x)} d x\right)-\lambda \int_{\Omega} f(x) \frac{\left|t \varphi_{0}\right|^{q(x)}}{q(x)} d x \\
& -\int_{\Omega} g(x) \frac{\left|t \varphi_{0}\right|^{r(x)}}{r(x)} d x-t \int_{\Omega} h(x) \varphi_{0} d x \\
\leq & \frac{m_{1} t^{\beta p^{+}}}{\left(p^{-}\right)^{\beta}}\left(\int_{\Omega}\left(\left|\nabla \varphi_{0}\right|^{p(x)}+\left|\varphi_{0}\right|^{p(x)}\right) d x\right)^{\beta}+\frac{\lambda t^{q^{+}}}{q^{-}} \int_{\Omega}|f(x)|\left|\varphi_{0}\right|^{q(x)} d x \\
& -\frac{t^{r^{-}}}{r^{+}} \int_{\Omega_{0}} g(x)\left|\varphi_{0}\right|^{r(x)} d x-t \int_{\Omega} h(x) \varphi_{0} d x .
\end{aligned}
$$

Therefore $I_{\lambda}\left(t \varphi_{0}\right) \rightarrow-\infty$ as $t \rightarrow+\infty$ since $1<q^{+}<\beta p^{+}<r^{-}$. So, for some $t_{0}$ large enough, $I_{\lambda}\left(t_{0} \varphi_{0}\right)<$ 0 . This ends the proof of Lemma 3.1.

Lemma 3.2. Assume $\left(M_{1}\right)-\left(M_{2}\right)$ and $\left(H_{0}\right)-\left(H_{2}\right)$. Then, $I_{\lambda}$ satisfies the Palais-Smale condition.

Proof. Let $\left\{u_{n}\right\} \subset W^{1, p(x)}(\Omega)$ such that

$$
I_{\lambda}\left(u_{n}\right) \rightarrow c, \quad I_{\lambda}^{\prime}\left(u_{n}\right) \rightarrow 0 \text { in }\left(W^{1, p(x)}(\Omega)\right)^{*}
$$


We claim that $\left\{u_{n}\right\}$ is bounded in $W^{1, p(x)}(\Omega)$. Suppose by contradiction up to subsequence $\left\|u_{n}\right\| \rightarrow+\infty$. Then, for $n$ large enough, it follows from $\left(M_{1}\right)-\left(M_{2}\right)$ and $\left(H_{1}\right)$ that

$$
\begin{aligned}
& c+1+|| u_{n}|| \\
\geq & I_{\lambda}\left(u_{n}\right)-\frac{1}{r^{-}}\left\langle I_{\lambda}^{\prime}\left(u_{n}\right), u_{n}\right\rangle \\
\geq & \widehat{M}\left(\int_{\Omega} \frac{\left|\nabla u_{n}\right|^{p(x)}+\left|u_{n}\right|^{p(x)}}{p(x)} d x\right) \\
& -\frac{1}{r^{-}} M\left(\int_{\Omega} \frac{\left|\nabla u_{n}\right|^{p(x)}+\left|u_{n}\right|^{p(x)}}{p(x)} d x\right) \int_{\Omega}\left(\left|\nabla u_{n}\right|^{p(x)}+\left|u_{n}\right|^{p(x)}\right) d x \\
& +\lambda \int_{\Omega}\left(\frac{1}{r^{-}}-\frac{1}{q(x)}\right) f(x)\left|u_{n}\right|^{q(x)} d x+\int_{\Omega}\left(\frac{1}{r^{-}}-\frac{1}{r(x)}\right) g(x)\left|u_{n}\right|^{r(x)} d x+\left(\frac{1}{r^{-}}-1\right) \int_{\Omega} h(x) u_{n} d x \\
\geq & \left(1-\frac{\theta p^{+}}{r^{-}}\right) \widehat{M}\left(\int_{\Omega} \frac{\left|\nabla u_{n}\right|^{p(x)}+\left|u_{n}\right|^{p(x)}}{p(x)} d x\right) \\
& -\lambda\left(\frac{1}{r^{-}}+\frac{1}{q^{-}}\right) \int_{\Omega}\left|f(x) \| u_{n}\right|^{q(x)} d x+\left(\frac{1}{r^{-}}-1\right) \int_{\Omega} h(x) u_{n} d x \\
\geq & \frac{m_{0}}{\left(p^{+}\right)^{\alpha}}\left(1-\frac{\theta p^{+}}{r^{-}}\right)|| u_{n}\left\|^{\alpha p^{-}}-\lambda\left(\frac{1}{r^{-}}+\frac{1}{q^{-}}\right) c_{1}\right\| f\left\|_{L^{q_{0}(x)}(\Omega)}\right\| u_{n} \|^{q^{+}} \\
& -c_{3}\left(1-\frac{1}{r^{-}}\right)\|h\|_{L^{s_{0}(x)}(\Omega)}|| u_{n} \| .
\end{aligned}
$$

Since $1<q^{+}<\alpha p^{-}$and $\theta p^{+}<r^{-}$, this last inequality is an absurd and hence $\left\{u_{n}\right\}$ is bounded in $W^{1, p(x)}(\Omega)$. Then, up to subsequence $u_{n} \rightarrow u$ in $W^{1, p(x)}(\Omega)$ and

$$
\int_{\Omega} \frac{\left|\nabla u_{n}\right|^{p(x)}+\left|u_{n}\right|^{p(x)}}{p(x)} d x \rightarrow l_{0}, \text { as } n \rightarrow+\infty .
$$

If $l_{0}=0$, then $\left\|u_{n}\right\| \rightarrow 0$. The proof is complete. Suppose that $l_{0}>0$ and prove that $\left\|u_{n}-u\right\| \rightarrow 0$. Indeed, we first note that

$$
\begin{aligned}
& M\left(\int_{\Omega} \frac{\left|\nabla u_{n}\right|^{p(x)}+\left|u_{n}\right|^{p(x)}}{p(x)} d x\right) \\
& \times\left[\int_{\Omega}\left(\left|\nabla u_{n}\right|^{p(x)-2} \nabla u_{n}-|\nabla u|^{p(x)-2} \nabla u\right) \nabla\left(u_{n}-u\right) d x+\int_{\Omega}\left(\left|u_{n}\right|^{p(x)-2} u_{n}-|u|^{p(x)-2} u\right)\left(u_{n}-u\right) d x\right] \\
& =\left\langle I_{\lambda}^{\prime}\left(u_{n}\right), u_{n}-u\right\rangle-M\left(\int_{\Omega} \frac{\left|\nabla u_{n}\right|^{p(x)}+\left|u_{n}\right|^{p(x)}}{p(x)} d x\right) \int_{\Omega}\left(|\nabla u|^{p(x)-2} \nabla u \nabla\left(u_{n}-u\right)+|u|^{p(x)-2} u\left(u_{n}-u\right)\right) d x \\
& +\lambda \int_{\Omega} f(x)\left|u_{n}\right|^{q(x)-2} u_{n}\left(u_{n}-u\right) d x+\int_{\Omega} g(x)\left|u_{n}\right|^{r(x)-2} u_{n}\left(u_{n}-u\right) d x+\int_{\Omega} h(x)\left(u_{n}-u\right) d x .
\end{aligned}
$$

Since $u_{n} \rightarrow u$, one has

$$
\left\{\begin{array}{l}
\left\langle I_{\lambda}^{\prime}\left(u_{n}\right), u_{n}-u\right\rangle \rightarrow 0 \\
\int_{\Omega}\left(|\nabla u|^{p(x)-2} \nabla u \nabla\left(u_{n}-u\right)+|u|^{p(x)-2} u\left(u_{n}-u\right)\right) d x \rightarrow 0
\end{array}\right.
$$


On the other hand, taking account the fact that $f \in L^{q_{0}(x)}(\Omega)$, for every $\varepsilon>0$, there exists $R_{\varepsilon}>0$ large enough such that $\Omega_{b} \subset B_{R_{\varepsilon}}$ and

$$
\|f\|_{L^{q_{0}(x)}\left(\Omega \backslash \Omega_{R_{\varepsilon}}\right)}<\varepsilon
$$

where $\Omega_{R_{\varepsilon}}=B_{R_{\varepsilon}} \backslash \Omega_{b}$. Since $f$ is bounded in $\Omega_{R_{\varepsilon}}$, Hölder's inequality and (3.10) imply

$$
\begin{aligned}
\int_{\Omega}\left|f(x) \| u_{n}-u\right|^{q(x)} d x \leq & \|f\|_{L^{\infty}\left(\Omega_{R_{\varepsilon}}\right)} \int_{\Omega_{R_{\varepsilon}}}\left|u_{n}-u\right|^{q(x)} d x+\int_{\Omega \backslash \Omega_{R_{\varepsilon}}}\left|f(x) \| u_{n}-u\right|^{q(x)} d x \\
\leq & \|f\|_{L^{\infty}\left(\Omega_{R_{\varepsilon}}\right)} \int_{\Omega_{R_{\varepsilon}}}\left|u_{n}-u\right|^{q(x)} d x \\
& +c_{0}\|f\|_{\left.L^{q_{0}\left(\Omega \backslash \Omega_{R_{\varepsilon}}\right.}\right)} \max \left(\left\|u_{n}-u\right\|_{L^{p^{*}(x)}\left(\Omega \backslash \Omega_{R_{\varepsilon}}\right.}^{q^{+}},\left\|u_{n}-u\right\|_{L^{p^{*}(x)}\left(\Omega \backslash \Omega_{R_{\varepsilon}}\right)}^{q^{-}}\right) \\
\leq & \|f\|_{L^{\infty}\left(\Omega_{R_{\varepsilon}}\right)} \int_{\Omega_{R_{\varepsilon}}}\left|u_{n}-u\right|^{q(x)} d x+c_{0} \varepsilon \max \left(\left\|u_{n}-u\right\|_{L^{p^{*}(x)}(\Omega)}^{q^{+}},\left\|u_{n}-u\right\|_{L^{p^{*}(x)}(\Omega)}^{q^{-}}\right) .
\end{aligned}
$$

According to the Sobolev compact embedding theorem in the bounded domain $\Omega_{R_{\varepsilon}}$, we see that $u_{n} \rightarrow u$ in $L^{q(x)}\left(\Omega_{R_{\varepsilon}}\right)$. Having in mind that $\left\{u_{n}\right\}$ is bounded in $L^{p^{*}(x)}(\Omega)$, we deduce from (3.11) that

$$
\int_{\Omega}|f(x)|\left|u_{n}-u\right|^{q(x)} d x \rightarrow 0, \text { as } n \rightarrow+\infty .
$$

In the similar way, we obtain

$$
\begin{aligned}
& \int_{\Omega} g(x)\left|u_{n}-u\right|^{r(x)} d x \rightarrow 0, \\
& \int_{\Omega} h(x)\left(u_{n}-u\right) d x \rightarrow 0 .
\end{aligned}
$$

Using again Hölder's inequality and (3.12)-(3.13), we could easily establish that

$$
\int_{\Omega} f(x)\left|u_{n}\right|^{q(x)-2} u_{n}\left(u_{n}-u\right) d x \rightarrow 0, \quad \int_{\Omega} g(x)\left|u_{n}\right|^{r(x)-2} u_{n}\left(u_{n}-u\right) d x \rightarrow 0 .
$$

By the virtue of the continuity of the function $M$, it follows from (3.7)-(3.9), (3.14) and (3.15) that

$$
\begin{aligned}
& M\left(\int_{\Omega} \frac{\left|\nabla u_{n}\right|^{p(x)}+\left|u_{n}\right|^{p(x)}}{p(x)} d x\right) \\
& \times\left[\int_{\Omega}\left(\left|\nabla u_{n}\right|^{p(x)-2} \nabla u_{n}-|\nabla u|^{p(x)-2} \nabla u\right) \nabla\left(u_{n}-u\right) d x+\int_{\Omega}\left(\left|u_{n}\right|^{p(x)-2} u_{n}-|u|^{p(x)-2} u\right)\left(u_{n}-u\right) d x\right] \\
& \rightarrow 0 .
\end{aligned}
$$

Hence

$$
\begin{aligned}
& \lim _{n \rightarrow+\infty}\left[\int_{\Omega}\left(\left|\nabla u_{n}\right|^{p(x)-2} \nabla u_{n}-|\nabla u|^{p(x)-2} \nabla u\right) \nabla\left(u_{n}-u\right) d x+\int_{\Omega}\left(\left|u_{n}\right|^{p(x)-2} u_{n}-|u|^{p(x)-2} u\right)\left(u_{n}-u\right) d x\right] \\
& =0 .
\end{aligned}
$$

Using the well known inequality in $\mathbb{R}^{N}$ given by

$$
\left[\left(|\xi|^{p-2} \xi-|\eta|^{p-2} \eta\right)(\xi-\eta)\right]^{\frac{p}{2}}\left(|\xi|^{p}+|\eta|^{p}\right)^{\frac{2-p}{2}} \geq(p-1)|\xi-\eta|^{p} \text { if } 1<p<2,
$$

and

$$
\left(|\xi|^{p-2} \xi-|\eta|^{p-2} \eta\right)(\xi-\eta) \geq 2^{-p}|\xi-\eta|^{p} \text { if } p \geq 2,
$$

we deduce from (3.16) that $\left\|u_{n}-u\right\| \rightarrow 0$. This completes the proof of Lemma 3.2. 
Proof of Theorem 2.4. In view of Lemmas 3.1, 3.2 and Mountain Pass theorem [23], there exists a weak solution $u_{1}$ of problem $\left(P_{\lambda}\right)$ with $I_{\lambda}\left(u_{1}\right)>0$. We now prove that there exists a second weak solution $u_{2} \neq u_{1}$. Choosing $\varphi_{1} \in C_{0}^{\infty}(\Omega)$ such that $\int_{\Omega} h(x) \varphi_{1} d x>0$. Then, by $\left(M_{1}\right)$, for small $t>0$,

$$
\begin{aligned}
I_{\lambda}\left(t \varphi_{1}\right) \leq & \frac{m_{1} t^{\beta p^{-}}}{\left(p^{-}\right)^{\beta}}\left(\int_{\Omega}\left(\left|\nabla \varphi_{1}\right|^{p(x)}+\left|\varphi_{1}\right|^{p(x)}\right) d x\right)^{\beta}+\frac{\lambda t^{q^{-}}}{q^{-}} \int_{\Omega}|f(x)|\left|\varphi_{1}\right|^{q(x)} d x \\
& +\frac{t^{r^{-}}}{r^{-}} \int_{\Omega} g(x)\left|\varphi_{1}\right|^{r(x)} d x-t \int_{\Omega} h(x) \varphi_{1} d x<0 .
\end{aligned}
$$

Thus for $\rho>0$ given in Lemma 3.1,

$$
-\infty<c_{\rho}:=\inf _{u \in \overline{B_{\rho}}} I_{\lambda}(u)<0, \quad \inf _{u \in \partial B_{\rho}} I_{\lambda}(u)>0,
$$

where $B_{\rho}$ is the ball centered at 0 and of radius $\rho$. Let us choose $0<\varepsilon<\inf _{u \in \partial B_{\rho}} I_{\lambda}(u)-\inf _{u \in B_{\rho}} I_{\lambda}(u)$. Using the above information, $I_{\lambda}: \overline{B_{\rho}} \rightarrow \mathbb{R}$ is lower bounded on $\overline{B_{\rho}}$ and $I_{\lambda} \in C^{1}\left(\overline{B_{\rho}}, \mathbb{R}\right)$. By Ekeland's variational principle [24], there exists $u_{\varepsilon} \in \overline{B_{\rho}}$ such that

$$
\left\{\begin{array}{l}
c_{\rho} \leq I_{\lambda}\left(u_{\varepsilon}\right) \leq c_{\rho}+\varepsilon \\
I_{\lambda}\left(u_{\varepsilon}\right)<I_{\lambda}(u)+\varepsilon\left\|u-u_{\varepsilon}\right\|, \quad u \neq u_{\varepsilon}
\end{array}\right.
$$

Since

$$
I_{\lambda}\left(u_{\varepsilon}\right) \leq \inf _{u \in \overline{B_{\rho}}} I_{\lambda}(u)+\varepsilon \leq \inf _{u \in B_{\rho}} I_{\lambda}(u)+\varepsilon<\inf _{u \in \partial B_{\rho}} I_{\lambda}(u)
$$

we can infer that $u_{\varepsilon} \in B_{\rho}$.

Now, we define $J_{\lambda}: \overline{B_{\rho}} \rightarrow \mathbb{R}$ by

$$
J_{\lambda}(u)=I_{\lambda}(u)+\varepsilon\left\|u-u_{\varepsilon}\right\| .
$$

It is easy to see that $u_{\varepsilon}$ is a minimum point of $J_{\lambda}$. It follows that

$$
\frac{J_{\lambda}\left(u_{\varepsilon}+t v\right)-J_{\lambda}\left(u_{\varepsilon}\right)}{t} \geq 0, \text { for } t>0 \text { small enough and } v \in B_{1} .
$$

It yields that

$$
\frac{I_{\lambda}\left(u_{\varepsilon}+t v\right)-I_{\lambda}\left(u_{\varepsilon}\right)}{t}+\varepsilon\|v\| \geq 0
$$

Letting $t \rightarrow 0$, we get

$$
\left\langle I_{\lambda}^{\prime}\left(u_{\varepsilon}\right), v\right\rangle+\varepsilon\|v\| \geq 0
$$

which implies $\left\|I_{\lambda}^{\prime}\left(u_{\varepsilon}\right)\right\|_{W^{1, p(x)}(\Omega)^{*}} \leq \varepsilon$. Therefore, we deduce that there exists a sequence $\left\{u_{n}\right\} \subset B_{\rho}$ such that

$$
I_{\lambda}\left(u_{n}\right) \rightarrow c_{\rho}, \quad I_{\lambda}^{\prime}\left(u_{n}\right) \rightarrow 0 \text { in } W^{1, p(x)}(\Omega)^{*} .
$$

Thanks to Lemma 3.2, up to subsequence $u_{n} \rightarrow u_{2}$ strongly in $W^{1, p(x)}(\Omega)$ with $I_{\lambda}\left(u_{2}\right)<0$. Therefore, we conclude that problem $\left(P_{\lambda}\right)$ admits at least two nontrivial solutions. This competes the proof of Theorem 2.4.

\section{Acknowledgement}

The authors are grateful to the reviewers for useful suggestions which improved the contents of this paper. 


\section{REFERENCES}

[1] V.V. Zhikov, Averaging of functionals in the calculus of variations and elasticity, Izv. Akad. Nauk SSSR Ser. Mat. 50 (1986), 675-710.

[2] M. Růžička, Flow of shear dependent electrorheological fluids, C. R. Acad. Sci. Paris 329 (1999), 393-398.

[3] M. Růžička, Electrorheological Fluids: Modeling and Mathematical Theory, Springer-Verlag, Berlin, 2002.

[4] M. Bocea, M. Mihăilescu, $\Gamma$-convergence of power-law functionals with variable exponents, Nonlinear Anal. 73 (2010), 110-121.

[5] M. Bocea, M. Mihăilescu, C. Popovici, On the asymptotic behavior of variable exponent power-law functionals and applications, Ric. Mat. 59 (2010), 207-238.

[6] Y. Chen, S. Levine, R. Rao, Variable exponent, linear growth functionals in image restoration, SIAM J. Appl. Math. 66 (2006) 1383-1406.

[7] S. Antontsev, S. Shmarev, Handbook of Differential Equations, Stationary Partial Differential Equations, vol. III, Chap. 1 (2006).

[8] M. Avci, R. Mashiyev, Existence and uniqueness of solutions of a nonlocal problem involving the $p(x)$-Laplacian, Annals of the University of Craiova, Mathematics and Computer Science Series, 1 (2014) 30-37.

[9] M. Avci, B. Cekic, R. A. Mashiyev, Existence and multiplicity of the solutions of the $p(x)$-Kirchhoff type equation via genus theory, Math. Methods Appl. Sci. 34 (2011), 1751-1759.

[10] X. Fan, On Nonlocal $p(x)$-Laplacian Dirichlet Problems, Nonlinear Anal. 72 (2010), 3314-3323.

[11] N.T. Chung, On some $p(x)$-Kirchhof type equation with weights, J. Appl. Math. Info. 32 (2014), 113-128.

[12] G. Dai, R. Ma, Solutions for a $p(x)$-Kirchhoff type equation with Neumann boundary data, Nonlinear Anal. Real Word Appl. 12 (2011) 2666-2680.

[13] G. Dai, X. Li, On nonlocal elliptic systems of $p(x)$-Kirchhoff-type under Neumann boundary condition, J. Math. Res. Appl. 33 (2013), 443-450.

[14] M. Massar, M. Talbi, N. Tsouli, Multiple solutions for nonlocal system of $(p(x), q(x))$-Kirchhoff type, Appl. Math. Comput. 242 (2014), 216-226.

[15] Z. Yucedag, M. Avaci, R. Mashiyev, On an elliptic system of $p(x)$-Kirchhoff-type under Neumann boundary condition, Math. Model. Appl. 17 (2012), 161-170.

[16] C. Chen, Y. Kuo, T. Wu, The Nehari manifold for a Kirchhoff type problem involving signchanging weight functions, J. Differential Equations 250 (2011), 1876-1908.

[17] C. Chen, J. Huang, L. Liu, Multiple solutions to the nonhomogeneous $p$-Kirchhoff elliptic equation with concave-convex nonlinearities, Appl. Math. Lett. 26 (2013), 754-759.

[18] J. Huang, C. Chen, Z. Xiu, Existence and multiplicity results for a $p$-Kirchhoff equation with a concave-convex term, Appl. Math. Lett. 26 (2013), 1070-1075.

[19] X. Fan, D. Zhao, On the spaces $L^{p(x)}(\Omega)$ and $W^{m, p(x)}(\Omega)$, J. Math. Anal. Appl. 263 (2001), 424-446.

[20] X. Fan, $p(x)$-Laplacian equations in $R^{N}$ with periodic data and nonperiodic perturbations, J. Math. Anal. Appl. 341 (2008), 103-119.

[21] X. Fan, X. Han, Existence and multiplicity of solutions for $p(x)$-Laplacian equations in $R^{N}$, Nonlinear Anal. 59 (2004), 173-188.

[22] S. Aouaoui, Existence and multiplicity results for some eigenvalue problems involving variable exponents, Nonlinear Anal. 80 (2013), 76-87.

[23] A. Ambrosetti, P. H. Rabinowitz, Dual variational methods in critical point theorey and applications, J. Funct. Anal. 14 (1973), 349-381.

[24] I. Ekeland, On the variational principle, J. Math. Anal. Appl. 47 (1974), 324-353. 